\title{
Dispositif de vision et modèles de pouvoir : « Devant la loi », de Kafka
}

\section{Maria Tortajada}

\section{(2) OpenEdition}

\section{Journals}

Édition électronique

URL : http://journals.openedition.org/ress/459

DOI : $10.4000 /$ ress.459

ISSN : 1663-4446

Éditeur

Librairie Droz

\section{Édition imprimée}

Date de publication : 1 janvier 2006

Pagination : 37-52

ISBN : 2-600-00959-0

ISSN : 0048-8046

\section{Référence électronique}

Maria Tortajada, «Dispositif de vision et modèles de pouvoir : « Devant la loi », de Kafka », Revue européenne des sciences sociales [En ligne], XLIV-133 | 2006, mis en ligne le 09 novembre 2009, consulté le 30 avril 2019. URL : http://journals.openedition.org/ress/459 ; DOI : 10.4000/ress.459 


\section{Maria TORTAJADA}

\section{DISPOSITIF DE VISION ET MODELLES DE POUVOIR : «EVANT LA LOI», DE KAFKA}

On peut tenter de définir la loi dans le texte célèbre de Kafka. Nous chercherons plutôt à décrire le fonctionnement du pouvoir. Pour saisir les implications de «Devant la loi », il est utile de passer par le révélateur cinématographique. Lorsqu'Orson Welles adapte Le Procès en 1962, il accorde une place prépondérante à ce texte célèbre que Franz Kafka avait intégré au manuscrit du roman. Dans le films, «Devant la loi» devient un prologue réalisé par Alexandre Alexeieff au moyen de sa technique d'animation: l'écran d'épingles. Welles n'en intègre pas moins la fameuse scène de la cathédrale (chapitre IX), transformant l'échange entre le prêtre et $\mathrm{K}$ en une sorte de duel où ce dernier, sous les traits d'Anthony Perkins, s'oppose à l'avocat, incarné par Welles lui-même' ${ }^{1}$. Welles-réalisateur fait en somme assumer le conflit à Welles-acteur, lui donnant comme «arme» un dispositif de vision: l'avocat raconte «Devant la loi», en projetant des images fixes tirées du film d'animation présenté dans le prologue ${ }^{2}$. Il piège momentanément $\mathrm{K}$ dans cette représentation, qui tantôt intègre l'ombre du jeune homme en silhouette, tantôt moule son visage, phagocytant son corps et transformant K en un personnage de la fable que l'avocat commente. La séquence devient ainsi un combat où $\mathrm{K}$ et l'avocat jouent de leur pouvoir, pouvoir qui leur est donné alternativement par leur capacité d'inscrire l'autre dans l'image projetée, de raconter soi-même l'histoire de «Devant la loi », de se rendre en somme maîtres de l'énonciation. Il s'agit de faire de l'autre son personnage ${ }^{3}$. Le projecteur, dispositif de vision spécifique, devient alors l'outil du pouvoir, et renvoie le spectateur, associé au point de vue de la caméra, à la place du démiurge. Ce choix de mise en scène rend compte d'une dimension essentielle de la parabole de Kafka, qui lie la question du pouvoir à la pratique des dispositifs de vision ${ }^{4}$.

Jouant magistralement des enchâssements et des ambiguïtés, le film transforme la figure du prêtre, dialoguant avec K, en une figure double: gardant une référence à la continuité, la scène présente d'abord l'abbé s'entretenant avec K dans l'église; elle montre ensuite la conversation dans un décor de structures métalliques (filmé dans la gare d'Orsay à Paris) où le prêtre (Michael Lonsdale) est devenu l'avocat (Orson Welles), avant de réapparaître sous les traits de Lonsdale pour clore la séquence. Enchâssement remarquable : l'avocat est le prêtre, qui est Welles-acteur, qui est Welles-réalisateur, qui, par le jeu des ombres devant la projection des images produites par l'écran d'épingles, devient le gardien de la porte.

2 Ce dispositif de vision de nature cinématographique renvoie aux pratiques du cinéma muet: un bonimenteur commentait le film rendant un spectacle visuel et sonore complet.

Le film fait de $\mathrm{K}$ un révolté et non pas seulement une victime.

${ }^{4}$ Le dispositif de vision est ce qui permet à un spectateur d'accéder à une représentation en fonction d'un certain «appareillage »: c'est-à-dire non seulement la machine de vision comme objet 
Différentes approches théoriques des dispositifs de vision ont envisagé ce rapport dans le courant des années 70, notamment autour de la question de l'idéo$\operatorname{logie}^{5}$. C'est le cas pour le dispositif cinématographique, dont la force illusionniste, considérée même parfois comme hallucinatoire, est chargée d'une valeur idéologique. Ces théories reprennent les analyses de la construction perspectiviste, que reproduit la camera obscura et font du spectateur un sujet centré: le lieu d'où toute la représentation s'organise, le centre du pouvoir et de la maîtrise sur le monde, dont la représentation obéit à une cohérence réaliste ${ }^{6}$. La caméra, comme l'appareil de projection, construisent un tel spectateur. Elles ajoutent aux effets de la camera obscura, des procédés proprement cinématographiques: le centrement du spectateur est ainsi obtenu grâce au champ contrechamp, de même que la continuité spatiale et temporelle qui construisent l'illusion d'un monde cohérent s'appuient sur tout un jeu de raccords, fondés sur l'identification du spectateur à la caméra ${ }^{7}$. Les aspects techniques (caméra, montage) sont abordés comme des indices dont la représentation témoigne. Mais parce qu'ils construisent la place du spectateur, ils touchent à la question des dispositifs.

La critique du pouvoir comme dispositif de regard a été, dans les années 70, un thème central des travaux de Michel Foucault. Son analyse du Panoptique de Bentham dans Surveiller et punir est devenu un modèle de référence en matière de dispositifs de vision ${ }^{8}$. Dans le panoptisme, le pouvoir est du côté du point de vue: l'assujettissement consiste à être vu, à devenir en quelque sorte transparent au

technique: par exemple, l'appareil de projection utilisé par Welles-avocat dans la scène de la cathédrale; mais aussi l'ensemble des éléments ou procédés mis en œuvre de la production à la monstration (au sens large du terme): ainsi, pour la photographie comme pour le cinéma, le processus chimique est un élément déterminant du dispositif. Si nous choisissons l'aborder ce texte sous cet angle c'est que nous travaillons sur la question des dispositifs de vision avec une visée épistémologique. Notre recherche actuelle nous conduit à travailler sur les cas précis de Henri Bergson, Etienne-Jules Marey et Alfred Jarry. Elle s'intègre dans un projet plus large, mené en collaboration avec François Albera à la Section d'histoire et esthétique du cinéma de l'Université de Lausanne, qui vise à définir ce que nous avons appelé «l'épistémè 1900 ». Voir F. Albera, M. Tortajada, «L'Epistémè «1900» », Le cinématographe, nouvelle technologie du XXe siècle/The Cinema, A New Technology for the 20th Century, Lausanne, Payot, 2004, pp. 45-62.

5 Voir notamment Sarah Kofman, Camera Obscura de l'idéologie, Galilée, 1973.

' Jean-Louis, Baudry, L'effet cinéma, Albatros, 1978. Les Cahiers du cinéma consacrent à ces question des articles de fond: Jean-Louis Comolli, «Technique et idéologie I», 229, mai 1971, pp. 419, «Technique et idéologie II », 230, juill. 1971, pp. 51-57, «Technique et idéologie III», 231, aoûtsept. 1971, pp. 42-49, «Technique et idéologie IV», 233, nov. 1971, pp. 39-45, «Technique et idéologie V», 234-235, déc. 1971, pp. 94-100; Pascal Bonitzer, «»Réalité » de la dénotation», 229, mai-juin 1971, pp. 39-41, «Le gros orteil. Réalité de la dénotation, 2 », 232, oct.1971, pp. 15-22, «Fétichisme de la technique: la notion de plan», 233, nov. 1971, pp. 4-10; Pascal Bonitzer et Serge Daney, «L'écran du fantasme. Les théories idéalistes du cinéma: André Bazin», 236-237, mars avril 1972, pp. 30-4; Jean-Pierre Oudart, «L'effet de réel », 228, mars-avril 1971, pp. 19-26, « Notes pour une théorie de la représentation» (suite de «L'effet de réel»), Cahiers du cinéma, 229, mai 1971, pp. 43-45, «Notes pour une théorie de la représentation» (suite et fin), 230, juill.1971, pp. 43-45.

Christian Metz, Le signifiant imaginaire, Bourgois, 1984 (1977).

'Il est essentiel à Foucault d'analyser dans la précision la forme du dispositif, qui est justement fonction de sa structuration de l'espace. Citons ici le début de ce passage bien connu et auquel le lecteur pourra se référer: «On en connaît le principe : à la périphérie un bâtiment en anneau; au centre, une tour; celle-ci est percée de larges fenêtres qui ouvrent sur la face intérieure de l'anneau; (...)» (Surveiller et punir, Paris, Gallimard (Tel), 1975, pp. 233-234). 
regard ${ }^{9}$. Pour Foucault, le pouvoir est désindividualisé et agit en quelque sorte automatiquement comme une «machinerie». Le sujet du regard en tant que tel ne détient pas ce pouvoir, c'est la place que tel sujet peut éventuellement occuper dans le dispositif de vision qui désigne le lieu où est situé le pouvoir. Celui-ci «fonctionne » parce que ce point de vue est toujours présent à l'esprit de celui qui se trouve, de ce fait, assujetti: "Un assujettissement réel naît mécaniquement d'une relation fictive. (...) Celui qui est soumis à un champ de visibilité, et qui le sait, reprend à son compte les contraintes du pouvoir; il les fait jouer spontanément sur lui-même $(\ldots)\rangle^{10}$. L'analyse du Panoptique démontre que son fonctionnement se fonde sur le quadrillage et la structuration architecturale de l'espace défini institutionnellement comme celui d'une prison.

Le pouvoir s'exerce donc grâce à l'agencement d'un point de vue, d'un objet de regard conscient d'être regardé et qui, de ce fait, se trouve assujetti. La relation que ces deux pôles entretiennent est fonction d'une certaine organisation de l'espace. Si, dans le Panoptique, le pouvoir est du côté du regard, celui-ci n'existe qu'en tant qu'il est lui-même construit par le dispositif entier. Même si le modèle de Bentham place au centre le regard, architecturalement, la machinerie du pouvoir que décrit Foucault produit un modèle décentralisé, un pouvoir disséminé où le centre est le produit des structures qui le constituent comme tel ${ }^{11}$. Viser le pouvoir en termes de «technologie», c'est, selon Foucault, se demander «par où passe le pouvoir plutôt que d'où il vient »; c'est mettre en lumière les relations le permettent $^{12}$. Ce dispositif exemplaire permet de comprendre l'assujettissement qui se généralise depuis l'âge classique et qui pénètre au cours du XIX Siècle $^{\mathrm{e}}$ toutes sortes d'institutions ${ }^{13}$.

L'analyse magistrale du Panoptique, avec les conclusions que Foucault en tire, est devenue un modèle très souvent repris, cependant, appliqué parfois à tout dispositif de vision dans les effets qu'il produit. En schématisant de telles positions, on pourrait dire qu'il suffit qu'il soit question de dispositifs de vision pour

9 «Il est vu mais il ne voit pas; objet d'une information, jamais sujet d'une communication» (ibid. p. 234).

${ }^{10}$ Ibid., p. 236.

${ }^{11}$ Le Panoptique: «Dispositif important, car il automatise et désindividudalise le pouvoir. Celuici a son principe moins dans une personne que dans une certaine distribution concertée des corps, des surfaces, des lumières, des regards; dans un appareillage dont les mécanismes internes produisent le rapport dans lequel les individus sont pris. Les cérémonies, les rituels, les marques par lesquels le plusde-pouvoir est manifesté chez le souverain sont inutiles. Il y a une machinerie qui assure la dissymétrie, le déséquilibre, la différence» (ibid., pp. 235-236).

${ }_{12}$ «Non pas: «D’où vient le pouvoir, où va-t-il?», mais: «Par où passe-t-il et comment cela se passe-t-il, quelles sont toutes les relations de pouvoir, comment est-ce qu'on peut décrire certaines des principales relations de pouvoir qui s'exercent dans notre société ?» (Michel Foucault, «Sexualité et pouvoir», conférence à l'université de Tokyo, débat, 1978, in Dits et écrits, II, 1976-1988, 2001, p. 567).

${ }^{13}$ Ainsi, Foucault aborde selon le modèle technologique le pouvoir exercé sur le corps de l'individu et sur les corps appartenant à tout un groupe social. Sur le bio-pouvoir, voir Histoire de la sexualité, I. La volonté de savoir, Paris, Gallimard, 1976. Notons que dans l'ouverture à son «archéologie de la représentation », Foucault produit également une analyse célèbre du pouvoir associée à un dispositif de vision: l'analyse des Ménines de Vélasquez dans Les mots et les choses (Paris, Gallimard, 1966). 
qu'il y ait assujettissement ${ }^{14}$, conclusion que ne se permet pas Foucault, puisqu'il analyse justement très précisément les conditions institutionnelles et structurelles impliquées.

La parabole ${ }^{15}$ de Kafka décrit un dispositif de vision et les pratiques de ce dispositif dans un contexte qui renvoie les personnages à la loi tout en mettant en scène des formes d'assujettissement. Elle propose une structure complexe du pouvoir, qui peut être analysée à partir des propositions théoriques de Foucault sans pour autant, nous semble-t-il, reproduire le modèle singulier du Panoptique. Nous tenterons donc de cerner un modèle de pouvoir en termes de machinerie ou de technologie. Gilles Deleuze et Felix Guattari ont fait déjà le lien entre l'œuvre de Kafka et l'analyse développée dans Surveiller et punir ${ }^{16}$. Ce qui nous frappe à la lecture de «Devant la loi », c'est que, les éléments de définition associés au Panoptique ne vont pas de soi: l'assujettissement n'est pas à priori d'être $v u$; le pouvoir ne se constitue pas à travers une position de maîtrise du regard; et la fable propose un dispositif de vision qui annule toute idée d'espace ${ }^{17}$. Etant donné la prédominance des modèles qui associent pouvoir et point de vue, la proposition de Kafka ne peut que nous intriguer.

\section{POUVOIR DE LA PAROLE}

«Devant la loi» de Kafka requiert qu'on s'arrête momentanément à son parcours éditorial. Publié séparément en $1915^{18}$, puis à l'intérieur du recueil de textes composé par Kafka, Un médecin de campagne ${ }^{19}$, il est partie intégrante du chapitre IX du Procès ${ }^{20}$. C'est bien dans la forme proposée au lecteur du roman

${ }^{14}$ Nous simplifions ici à l'extrême certaines analyses de Jonathan Crary (L'art de l'observateur. Vision et modernité au XIXe siècle, Nîmes, Jacqueline Chambon, 1994), qui par ailleurs formule une proposition particulièrement intéressante. Nous avons exprimé nos réserves de manière plus étendue et nuancée dans «Archéologie du cinéma: de l'histoire à l'épistémologie» (Cinémas «Histoires croisées des images: Objets et méthodes », (dir. Edouard Arnoldy), vol. 14, nos 2-3, printemps 2004, pp. 19-52).

${ }^{15}$ Nous désignerons «Devant la loi» par des synonymes comme «fable», «parabole», «histoire», pour leur référence commune à la notion de «récit», et malgré les connotations différentes de ces termes.

${ }^{16}$ Kafka. Pour une littérature mineure, Paris, Minuit, 1975, p. 103.

${ }^{17}$ Soulignons ici l'intérêt de l'adaptation du Procès réalisée par Welles qui, justement, construit une vision spatialisée des rapports de pouvoir tout au long du film.

${ }^{18}$ Vom jüngsten Tag. Ein Almanach neuer Dichtung, daté de 1916, mais paru dès 1915. Voir Franz Kafka, Euvres complètes, III, Paris, (éd. Claude David), Gallimard (La Pléiade), 1984, p. 1500 (note 3 de la p. 743).

19 Recueil de récits paru en 1919 qui comporte «Devant la loi». En 1917, Kafka propose un plan du recueil à son éditeur où figure ce même titre, qui n'apparaîttra pas dans le roman publié («Lettre à Kurt Wolf, Prague le 20 août 1917 », Euvres complètes, III, ibid., p. 782).

${ }^{20}$ L'édition du Procès établie, comme on le sait, par Max Brod a été publiée de manière posthume. Elle a été remise en question quant à l'ordre des textes par H. Uyttersprot («Eine neue Ordnung der Werke Kafkas? Zur Struktur von Der Prozess und Amerika», Antwerpen, 1957), ce qui a soulevé une polémique. Voir à ce propos Hartmut Binder, Kafka-Kommentar, II, Zu den Romanen, Rezensionen, Aphorismen und zum Brief an den Vater, II, München, Winkler Verlag, 1976, pp. 160- 
que nous allons l'aborder. Car le début et la fin du chapitre IX soulignent aussi bien les effets du pouvoir mis en scène dans la fable que certains de ses ressorts. La controverse entre le prêtre et $\mathrm{K}$ en témoigne: la fin du chapitre ne va cesser d'enchaîner différentes «interprétations» de «Devant la loi », différentes variantes, articulant toujours des thèses contraires ${ }^{21}$. Tous les arguments s'annulent, il n'y a pas de vérité, de même qu'il n'y a pas d'énonciateur «responsable» ou «garant» d'une vérité. Le prêtre, malgré sa fonction, ne cessera de répéter devant $\mathrm{K}$, sous différentes formes:

\begin{abstract}
Ne te méprends pas à mes paroles, répondit l'abbé. Je me contente d'exposer les diverses thèses en présence. N'attache pas trop d'importance aux gloses. L'Ecriture est immuable et les gloses ne sont souvent que l'expression du désespoir que les glossateurs en éprouvent $t^{22}$.
\end{abstract}

Ceci ne l'empêchera pas de multiplier les versions: c'est l'ironie du texte de $\mathrm{Kafka}^{23}$. Nous ne pouvons pas ne pas être concernés par cette pointe, qui renvoie tout lecteur à un processus d'échec s'il part à la recherche de la «vérité» de la fable. Dans ce sens, il vaudrait mieux se contenter de rire à la lecture de «Devant la loi », d'un rire grinçant néanmoins ! ?4 $^{24}$

Nous restons cependant rivés à la parabole comme l'homme qui, dans la fable, ne s'éloigne pas de la porte. Les variantes qu'énonce l'abbé devant K s'enchâ̂nent, captant l'attention du lecteur. Toutes prennent la fable très au sérieux en considérant les motivations des personnages: leur action est appréciée selon des critères de morale et suivant des analyses qui s'attachent au comportement, au caractère, à l'intention du gardien tout particulièrement, mais aussi de l'homme. Il s'agit de cerner leur rapport à la loi cachée derrière la porte car, si la vérité existe, c'est en référence à elle. Cette frénésie d'interprétations peut être considérée comme un effet du pouvoir que déploie ce texte. En témoigne, à un autre niveau, la bibliographie abondante sur le texte de Kafka. Nous n'y échappons pas. Reste peut-être une alternative: au lieu de viser la loi, ou son représentant, nous nous concentrerons sur le fonctionnement du pouvoir compris comme technologie. Le pouvoir exercé sur le lecteur a à voir avec celui que met en scène la fable.

Malgré l'ironie, la parabole nous prend aux sentiments - disons, au sentiment du tragique. Ainsi commence l'histoire ${ }^{25}$ :

194, et Claude David, Euvres complètes, I, Paris, Gallimard (La Pléiade), 1976, pp. 967-972. Gilles Deleuze et Felix Guattari (op. cit.), retiennent cette remise en question contestée. Cependant, leur ouvrage est particulièrement intéressant pour son approche globale de l'œuvre de Kafka.

${ }^{21}$ «- Tu touches ici à la thèse opposée, lui dit l'abbé», (F. Kafka, Le Procès Paris, Gallimard (Folio), 1987 (1933)., p. 271)

22 Ibid., p. 268.

${ }^{23}$ Ainsi, notamment: «(...) bien des exégètes s'étonnent» (p. 266), «Les glossateurs disent à ce propos (...)», (p. 267), «(...) bien des gens vont même plus loin et disent (...)» (p. 268), « On dit, (...); d'autres disent (...)» (p. 268); «Les glossateurs n'y voient d'ailleurs rien de surprenant (...)» (p. 270), ibid.. Nous sommes presque dans la parodie de la controverse.

${ }^{24}$ A ce sujet, les lignes de Deleuze et Guattari nous paraissent particulièrement intéressantes (op. cit., pp. 75-77).

${ }_{25}$ Pour les citations de «Devant la loi », nous renvoyons le lecteur au Procès, op. cit., pp. 263-265. 
Une sentinelle se tient postée devant la Loi; un homme [de la campagne] vient un jour la trouver et lui demande la permission de pénétrer [dans la Loi]. Mais la sentinelle lui dit qu'elle ne peut pas le laisser entrer en ce moment.

L'homme restera devant cette porte durant toute sa vie. Ce n'est qu'à la fin de ses jours qu'il pose une dernière question à la sentinelle:

«- Si tout le monde cherche à connaître la Loi, dit l'homme, comment se fait-il que personne d'autre que moi ne t'ait demandé d'entrer?» Le gardien voit que l'homme est sur sa fin et, pour atteindre son tympan mort, il lui rugit à l'oreille: «Personne que toi n'avait le droit d'entrer ici, car cette entrée n'était faite que pour toi, maintenant je pars, et je ferme [la porte].»

La parole du gardien est décisive. Elle encadre toute l'histoire entre deux actes d'autorité: la sentinelle signifie à l'homme un interdit pour finalement formuler ce qui était son droit singulier, droit que l'homme a justement ignoré.

A la fin du récit, le lecteur ne peut s'empêcher d'éprouver intensément que l'homme a manqué quelque chose: il est resté devant cette porte alors qu'il aurait pu entrer; il a passé sa vie à attendre, et l'a en quelque sorte perdue. Ce gâchis ne peut qu'ébranler le lecteur comme il révolte $\mathrm{K}$ : «Le gardien a donc trompé l'homme, dit aussitôt K que l'histoire avait vivement intéressé» (p. 265).

Si la loi est donnée d'emblée comme la question posée aux personnages et au lecteur, le terme de pouvoir n'est jamais nommé: c'est pourtant lui qui détermine l'action, structurée d'emblée par ces deux interventions de la sentinelle. Celle-ci exerce un pouvoir qui obéit à un mécanisme juridique: il dit non, il interdit ${ }^{26}$. C'est le pouvoir hiérarchisé, clairement situé, qui se donne comme censeur. Une forme traditionnelle du pouvoir, articulée à une loi transcendante, inatteignable, dont le gardien est le représentant. C'est le fonctionnement que Foucault oppose au pouvoir moderne, technologique, organisé selon la norme et suivant une logique disciplinaire, pourvoir intériorisé par celui qui s'y soumet. L'œuvre de Kafka a été lue comme une mise en crise des formes du pouvoir traditionnel, dont le modèle judaïque archaïque est le référent ${ }^{27}$. Pour Ulf Abraham, «Devant la loi » construit l'image de la Loi archaïque, qui distingue ses sujets, singulière pour chacun et non pas égale pour tous. La sentinelle l'affirme finalement devant l'homme vieilli: la porte n'était là que pour lui. La fable raconte en somme l'inadéquation de la demande de l'homme face à cette loi de la tradition, qui ne fonctionne plus: la sentinelle, en définitive, ne représente qu'elle-même ${ }^{28}$.

${ }^{26}$ Voir par exemple: «Les rapports de pouvoir passent à l'intérieur des corps », Entretien avec L. Finas, La Quinzaine littéraire, janvier 1977, in Michel Foucault, Dits et écrits, II, op. cit., pp. 228229.

${ }^{27}$ Ulf Abraham décrit notamment en des termes foucaldiens le pouvoir normalisé et disciplinaire qui vient se confronter, chez Kafka, à des modèles archaïques hérités du droit judaïque (Der Verhörte Held. Recht und Schuld im Werk Franz Kafkas, Münche, Wilhelm Fink Verlag, 1985, tout particulièrement pp. 126-139).

${ }^{28}$ Ibid., pp. 116-119, 129. François Ost, dans son récent ouvrage, développe également une analyse intéressante du fonctionnement de la loi dans l'œuvre de Kafka, décrivant, pour la parabole qui nous intéresse ici, une loi archaïque dans une «société désinstituée» (Raconter la loi. Aux sources de l'imaginaire juridique, Odile Jacob, 2004, voir notamment pp. 385-387). 
Pourtant, si l'homme s'arrête devant l'interdit, et si l'histoire continue, racontant sa vie devant cette porte, c'est bien que la parole performative de la sentinelle est suivie d'un effet. C'est bien que le garde a parlé au nom de la Loi. Si ce portier apparaît comme une instance de pouvoir, c'est qu'il tient ce pouvoir de son lien avec la Loi dont il est le serviteur ou le représentant, selon les interprétations. La Loi inatteignable et transcendante est ce qui légitime ce pouvoir: on ne passe pas la porte, on ne peut l'atteindre.

Cette forme de domination archaïque agit sur un point essentiel: sur le désir de l'homme d'entrer dans la Loi; elle est d'autant plus efficace que la Loi demeure inatteignable, même si la porte reste ouverte. Mais l'obsession de pénétrer dans l'espace de la loi, de trouver ce qui se cache, de satisfaire à l'interrogation de la métaphore et du symbolique à laquelle le lecteur n'échappe pas, appelé en somme à reconstruire un sens pour cette Loi invisible, nous détourne d'un aspect essentiel: c'est-à-dire de tout ce qui est exposé par le texte de manière beaucoup plus prosaïque: un dispositif de vision arrimé à des actes verbaux de pouvoir. Si la fable présente la porte comme une entrée que ni l'homme, ni le garde, ni le lecteur ne traverseront jamais, il ne cesse de nous montrer cette porte comme un point de vue à plusieurs usages. Notre hypothèse est que ce texte parle d'une forme de pouvoir qui se comprend à partir du moment où on le vise comme une machinerie fondée sur un dispositif de vision, qui, paradoxalement, relève d'un modèle archaïque.

La place essentielle accordée par Kafka à la question du point de vue ne devrait pas nous surprendre, puisque tout le comportement de $\mathrm{K}$, dès son arrivée dans la cathédrale au début du chapitre IX, est déterminé par son rapport à des dispositifs de vision. K entre seul dans l'édifice plongé dans l'obscurité. Son activité première est l'observation, guidée notamment par un jeu de lumières: des chandelles isolées ou «un grand triangle de flammes de cierges » (p. 253) attirent son regard ${ }^{29}$. La cathédrale est appréhendée comme un musée et non pas comme un espace sacré: K se présente avec un «recueil de photographies» (p. 252) à la main - nous apprendrons plus tard qu'il s'agit d' «un album des curiosités de la ville» (p. 260). Il est là pour faire visiter ce monument à un client italien. C'est pourtant seul qu'il va parcourir l'espace, faisant des haltes, comme pour les étapes d'une exposition. Les difficultés de l'observation sont sans cesse soulignées ${ }^{30}$. A chaque fois, le texte précise le type de regard jusque dans les détails. En somme, le parcours de la cathédrale se présente comme d'une série d'épreuves confrontant un sujet à des dispositifs divers: une cathédrale-musée avec ses œuvres d'arts, un album de photographies ${ }^{31}$. «Devant la loi », point culminant du chapitre, exacerbe le phénomène mettant à l'épreuve des personnages face à un dispositif de vision minimal.

\footnotetext{
${ }^{29}$ Par ailleurs, K est muni d'une «lampe de poche» (op. cit., p. 260).

${ }^{30}$ «L'Italien avait donc agi avec autant de discernement que d'impolitesse en ne venant pas; il n'aurait rien pu voir. On aurait été obligé de se contenter d'explorer quelques statues pouce par pouce avec la lampe de poche de K» (ibid., p. 254). C'est bien ce que K entreprend.

${ }^{31}$ Jusqu'à la mise en abyme de l'activité de spectateur par la statue d'un bas-relief: un grand chevalier «semblait observer attentivement une scène qui devait se passer devant ses yeux » (ibid., p. 254).
} 


\section{LE DISPOSITIF DE VISION}

Entrer dans la loi: là réside l'action dont il est question dans cette parabole. Pourtant, tout cela pourrait ne pas avoir de sens: peut-on en effet autrement que sous forme métaphorique ou symbolique «entrer dans la loi»? On a vite fait de constater que l'homme ne passe pas la porte: c'est l'effet immédiat de l'interdit. Ce dont il s'agira alors, c'est de regarder à travers elle. Plutôt qu'un passage, cette entrée et son garde sont les deux pièces d'un dispositif de vision. La parabole en son entier est un grand mécanisme articulant ensemble plusieurs pratiques du premier dispositif minimal.

I. On le découvre à travers le premier usage qui en est fait. Après le refus essuyé par l'homme, le texte continue:

La sentinelle s'efface devant la porte, ouverte comme toujours, et l'homme se penche pour regarder à l'intérieur [à travers la porte]. (p. 263)

La porte, telle une fenêtre, présente un cadre minimal du regard et désigne ainsi un point de vue que la fable s'empresse de mettre en place. C'est un topos que le réalisme à fort souvent utilisé pour définir le lieu du regard. La sentinelle apparaît alors comme «l'obturateur» potentiel de cette ouverture, qui justement n'a pas de porte capable de la fermer. Ce qu'il y a à voir, ou que l'on pourrait espérer voir, c'est la Loi. Mais on ne saura jamais ce que l'homme a vu. Pourtant, ce qui a été posé sans recours à une rhétorique du symbole est que la porte et son gardien fonctionnent comme un dispositif de vision.

II. Le garde fournit immédiatement une autre description de ce dispositif:

La sentinelle, le voyant faire, rit et dit: «Si tu en as tant envie essaie donc d'entrer malgré ma défense. Mais dis-toi bien que je suis puissant. Et je ne suis que la dernière des sentinelles. Tu trouveras à l'entrée de chaque salle des sentinelles de plus en plus puissantes; dès la troisième, même moi je ne peux plus supporter leur vue.

Le portier est doublement défini comme point de vue. Il se pose d'abord en observateur de l'homme: le voyant faire, et riant de ce spectacle, il se met à lui parler. Malgré l'affirmation renouvelée de l'interdit et la mise au défi de le transgresser, qui sont autant de rappels du premier exercice de son pouvoir, la mention de la vue par le gardien («je ne peux plus supporter leur vue») fait entrer ensuite ses paroles dans la série des éléments qui construisent un nouvel usage du dispositif de vision. La particularité ici est que le gardien fait part à l'homme de son utilisation du dispositif sans l'essayer devant lui. Si les paroles du garde sont avant tout descriptives («Tu trouveras à l'entrée...»), la fin de sa phrase mentionne son expérience propre, ce qui présuppose un récit implicite et donc une action essentielle: regarder les sentinelles, utiliser le dispositif de vision. Par sa proto-narration, le portier se présente comme un témoin digne de foi, et donc comme un modèle.

Le terme de «vue» est d'autant plus important qu'il est ambigu ${ }^{32}$ : il y a là comme un tremblement du discours qui doit retenir l'attention du lecteur. La vue,

${ }^{32}$ L'ambiguïté est là aussi en allemand: «Von Saal zu Saal stehen aber Türhüter einer Mächtiger als der Andere. Schon den Anblick des Dritten kann nicht einmal ich ertragen» (REF). 
c'est le regard. C'est le regard sur le portier de ces autres sentinelles; mais la vue est aussi le spectacle qu'offrent ces sentinelles au premier portier, instauré en point de vue et leur faisant face. Le garde est bien en train de «raconter» qu'il est lui-même l'un des utilisateurs du dispositif de vision. Peut-être est-il entré? On ne peut que spéculer sur la question ${ }^{33}$. Ce que le texte indique explicitement, c'est qu'il est un point de vue dans une relation qu'on peut qualifier de spéculaire: il regarde un garde, son semblable, un garde qui est sa propre image de garde et qui le regarde en retour. Or, cette figure se répète au moins trois fois: dès la troisième sentinelle, il ne peut plus faire face. Ce qui lui est alors insupportable, c'est la répétition des regards, ou leur nombre. Cette limite à son expérience comporte comme implicite que d'autres portiers se tiennent au-delà, défendant d'autres portes, et ainsi jusqu'à l'infini.

Cet usage du dispositif premier renvoie la loi à l'infini de la représentation, qui dans une approche symbolique peut devenir le tenant lieu de l'Infini, alors chargé du sens plein de la transcendance. Mais, de fait, pour le lecteur de Kafka, pour l'homme qui écoute, comme pour le garde qui raconte, s'il y a quelque chose $\grave{a}$ voir dans cette proto-narration, c'est la répétition du premier dispositif: porte plus sentinelle, dans un jeu d'enchâssements. L'infini peut être un «lieu» qui convient bien à la transcendance, donnant forme à ce trait qui fait de la loi l'inatteignable; mais ce point géométrique construit par un dispositif de vision où se répètent les regards et les portes, est construit par un système de reflets: un cadre dans un cadre, infiniment reproduits, c'est ce que l'on voit dans un miroir quand un autre miroir lui fait face, désignant à la fois le point de vue et le point de fuite dans la glace. Parfaite figure de la tautologie: si derrière la porte, à l'infini, se trouve la Loi, elle est à l'image du sujet qui lui fait face et qui prend place devant le miroir. La Loi, en somme, c'est ce que le sujet projette en elle: son désir, dira une lecture psychanalytique, un Dieu transcendant, comme semble l'impliquer le ton pour le moins biblique et la forme de la parabole ${ }^{34}$. Mais la figure spéculaire renvoie à l'illusion, au leurre. Mentionner le miroir, c'est déjà construire la critique de la transcendance, de ce garant du pouvoir archaïque. C'est dire que la Loi n'a donc pas d'existence en elle-même: elle est un fantôme du sujet. Cependant, la machinerie du pouvoir que développe la fable ne s'arrête pas au modèle spéculaire que présente le gardien. La Loi transcendante et la critique du pouvoir qui s'y réfère ne sont que les pièces de la machine.

Il faut remarquer que le dispositif que décrit le portier introduit une précision quant à la focalisation. Alors qu'on pourrait penser que le but de celui qui regarde est de voir derrière chaque porte, de contempler la Loi - c'est l'hypothèse du

\footnotetext{
${ }^{33}$ C'est d'ailleurs une des variantes interprétatives exposées par le prêtre à la fin du chapitre IX du Procès. Toute hésitation concernant la vérité de la parole du garde nous entraîne vers la spéculation. Que le garde dise vrai ou faux ne change rien au fait que sa proto-narration présente un usage du dispositif qui entraîne certains effets de pouvoir. Il y a quelque chose de performatif dans cette parole: une fois qu'il a décrit ce dispositif, sa propre expérience de point de vue face à la porte de la loi, le garde oblige celui qui l'écoute à se situer par rapport à sa proposition. C'est bien ce qu'expose la fable.

${ }^{34}$ De la référence biblique ou talmudique, nous ne dirons rien. Les commentaires de l'œuvre de Kafka qui prennent cette voie son très nombreux. Citons Gerhard Isermann, (Unser Leben, unser Prozess, Theologische Fragen bei Kafka, Wuppertal, 1969), qui traite de l'influence de l'Ancien Testament sur les récits de Kafka.
} 
dispositif spéculaire: l'objet du regard pourtant invisible est bien l'infini de la Loi -, le portier, lui, observe les autres gardiens, transformant ces derniers en objet de regard. Ce faisant, il fournit à l'homme un mode d'emploi du dispositif premier: en regardant à travers la porte, on expérimente la vue sur les sentinelles, disposées en série.

III. Regarder la sentinelle plutôt que la Loi, c'est justement ce que va faire l'homme de la fable: il va observer un garde, celui qui est le plus près de lui. Il va même y consacrer sa vie. Le passage des ans est pris en charge par une nouvelle expérience du regard, une troisième utilisation du dispositif premier. Le texte encadre d'ailleurs cette partie de l'histoire par la mention du regard:

L'homme [de la campagne] ne s'était pas attendu à de telles difficultés, il avait pensé que la Loi devait être accessible à tout le monde et en tout temps, mais maintenant, en observant mieux la sentinelle, son manteau de fourrure, son grand nez pointu et sa longue barbe rare et noire à la tartare, il se décide à attendre quand même jusqu'à ce qu'on lui permette d'entrer. (nous soulignons)

Les années s'écoulent et l'homme renouvelle les tentatives de passer la porte; mais l'essentiel est que:

\begin{abstract}
Pendant ses longues années d'attente, l'homme ne cesse presque jamais d'observer la sentinelle. Il en oublie les autres gardiens, il lui semble que le premier est le seul qui l'empêche d'entrer dans la Loi. (...) Il retombe en enfance, et comme, au cours des longues années où il a étudié la sentinelle, il a fini par connaître jusqu'aux puces de son col de fourrure, il prie les puces elles-mêmes de l'aider à fléchir le gardien. (nous soulignons)
\end{abstract}

Le regard scrutateur impliqué par cette «étude», qui s'attarde aux puces cachées dans le col du gardien, pourrait être une pointe ironique: l'homme, s'attachant à l'inessentiel, à ce détail infime que sont les petits insectes, manque la grandeur de la Loi. Mais les puces soulignent surtout de manière aiguë l'activité de cet observateur, car elles renvoient à un regard presque impossible. Elle rappellent un type de spectacle courant au tournant du $\mathrm{XIX}^{\mathrm{e}}$ et $\mathrm{XX}^{\mathrm{e}}$ siècles, qui joue sur un paradoxe. Les dresseurs de puces, dans les foires, choisissent justement d'exhiber ce petit animal que l'on ne peut voir que difficilement. Ce n'est pas un hasard si le cinéma s'est intéressé aux montreurs de puces: citons l'un des plus célèbres, Les feux de la rampe (Limelight, 1952) où Chaplin, dans le rôle de Calvero, prétend, du haut de la scène d'un théâtre, exhiber ces parasites. Thomas Koerfer construira tout un film sur ce type de représentation dans La mort $d u$ directeur du cirque de puces (Der Tod des Flohzirkusdirektors oder Ottocaro Weiss reformiert seine Firma, 1973). Avec la mention des puces, le texte de Kafka s'inscrit lui aussi dans le contexte hérité du XIX ${ }^{\mathrm{e}}$ siècle, faisant du regard l'un des fils conducteurs du récit. D'ailleurs, le narrateur enchaîne encore sur la question du regard:

Finalement, sa vue s'affaiblit et il ne sait si la nuit se fait vraiment autour de lui ou s'il est trompé par ses yeux. Mais maintenant il discerne dans l'ombre l'éclat d'une lumière qui brille [inextinguiblement] à travers les portes de la Loi.

En somme, dans la troisième expérience du dispositif, l'homme fait comme la sentinelle: il fixe un garde. Il a beau renouveler sa demande en essuyant les refus de cet intermédiaire, il concentre son intérêt sur lui. Il adopte ainsi le modèle que 
lui avait proposé la sentinelle lorsqu'elle lui avait décrit sa propre expérience; mais l'homme l'adapte à son propre usage car, à la différence de celui qui est devenu son interlocuteur premier, il détourne ses yeux de la porte pour observer la seule sentinelle qui se trouve devant lui : ce faisant, il échappe à la structure spéculaire exposée dans la fable. Imitation et réappropriation du modèle ont conduit l'homme à «négliger» le lieu de la transcendance, désirant toujours entrer dans la Loi, mais «oubliant» d'y porter son regard.

IV. Le dernier enchaînement du texte met donc à nouveau l'accent sur la vue cette fois celle de l'homme, qui s'affaiblit - ouvrant à un dernier usage du dispositif de vision premier. Alors advient ce grand moment qui peut apparaître comme une petite révélation: la lumière se donne à voir à travers la porte de la Loi - sans qu'aucun effort volontaire ne soit requis de l'homme et au moment où ses capacités visuelles sont défaillantes.

La lumière que l'homme perçoit est d'abord la matière qui fait fonctionner tout dispositif de vision. Ce n'est pas rien dans le contexte de cette fable. Cette expérience souligne le lien entre la loi et les pratiques du regard. On pourrait faire aussi de cette lumière le symbole de la transcendance, «la Lumière divine ». Non sans ironie, la fable vient semer le doute à ce sujet, car l'homme a pu tout aussi bien être «trompé par ses yeux». Si dans le modèle spéculaire le Sens était miné par l'effet du miroir qui situe le sujet à la place même de la Loi en renvoyant l'un et l'autre à la tautologie, dans cette dernière expérience, le regard de l'homme, tout occupé d'abord à contempler les puces, est sollicité par une représentation qui joue culturellement avec la transcendance. Mais encore une fois, comme avec le miroir. L'illusion est un possible du dispositif de vision. On constate que dès que la fable fait jouer de la transcendance elle introduit des indices qui toute certitude.

\section{MODÈLES DE POUVOIR}

A l'issue de ces quatre pratiques du dispositif de vision premier et minimal, la porte et son gardien, la fable revient à une parole de pouvoir explicite: nous l'avons vu, après avoir posé l'interdit qui inaugurait la parabole et forçait l'homme à attendre, le garde formule un droit. A cette position d'autorité exhibée, il faut en ajouter une autre qui détermine au moins autant le comportement de l'homme: le garde propose un modèle de regard. Pour ce faire, il use doublement du dispositif de vision: il le «raconte», disant l'avoir expérimenté. Le pouvoir du gardien n'agit pas par un jeu d'ordre ou d'interdit mais par l'exemple qu'il donne. Pour l'homme, il ne s'agit plus d'obéir, mais d'imiter. Dans la seconde forme verbale de l'exercice du pouvoir, la proto-narration, la porte n'est plus considérée comme une entrée, mais comme un élément du dispositif de vision, décrit par le gardien comme une représentation spéculaire. Le pouvoir qui s'exerce par l'exemple peut être considéré comme une «suggestion» à laquelle l'homme peut obéir ou non. D'ailleurs, nous avons vu qu'il suit le modèle présenté en se l'appropriant et, pouvons-nous croire, exerçant quelque peu sa liberté: durant tout sa vie, il va observer la sentinelle; la porte n'attirera son regard à nouveau que peu avant sa fin. Il fait sans doute preuve de myopie mais, il évite néanmoins le piège spéculaire. Pourtant, la souplesse apparente de la suggestion ne change pas la 
forme du pouvoir. Ce que nous décrivons ici reste encore un modèle de domination unilatérale, où le garde, représentant de la loi, a somme toute changé de tactique, ou articulé une approche à une autre ${ }^{35}$.

Est-ce donc que la mise en évidence des usages des dispositifs de vision nous conduit à constater le même fonctionnement du pouvoir hiérarchique, à travers une variante adoucie? En somme, si ce dispositif se distingue du Panoptique de Foucault, n'est-ce pas justement parce qu'il donne à voir une représentation du pouvoir archaïque, dont nous avons pu mentionner ici certains indices: la loi transcendante, la formulation de l'interdit et du droit singulier par le garde, son représentant, qui use également de l'autorité de l'exemple pour mieux asseoir son pouvoir? L'alliance du dispositif de vision et de la suggestion par l'exemple serait la version atténuée du pouvoir brutal, qui interdit l'entrée en ignorant la question du regard.

A l'inverse des procédures disciplinaires dont le Panoptique est le parangon, le pouvoir traditionnel s'exhibe dans les fastes qui confirment son autorité. Ainsi, dans la fable, la Loi est placée à l'horizon du dispositif spéculaire construit par la narration du portier, objet de regard par excellence, qui, même s'il reste non vu, convoque tous les ressorts de la représentation pour son exhibition, et occupe le lieu le plus important de la structure spéculaire: le point de fuite par rapport auquel les regards s'organisent ${ }^{36}$. Dans cette perspective, le garde, comme objet de regard, est encore un relais du pouvoir. Nous voici donc conduits à interroger le sens qui se cache dans ce point de fuite et qui paraît incontournable. Or si, pour définir le pouvoir, nous sommes contraints de rechercher la nature de la Loi c'est-à-dire «d'entrer dans la Loi » ou de nous mirer dans le dispositif spéculaire qui nous est présenté -, c'est que nous sommes encore une fois détournés de son fonctionnement et occupons la même place que les personnages de la fable. Si nous en restons là, nous devons conclure que le pouvoir mis en place par cette parabole appartient bien au modèle archaïque. L'analyse des usages du dispositif de vision nous montre pourtant autre chose: c'est que la Loi n'est qu'un élément d'un mécanisme plus vaste.

Pour éviter une interrogation qui nous renvoie toujours à la transcendance, il est utile de saisir le fonctionnement du pouvoir à travers ses effets. Cela revient à se demander comment la structure complexe de la parabole, qui articule plusieurs usages du dispositif de vision et différents actes de parole, parvient à produire de l'assujettissement.

I. Lorsque le garde interdit le passage, l'effet de cet exercice du pouvoir que nous disons traditionnel se mesure à l'obéissance: l'homme ne passe pas. Il aurait

${ }^{35}$ Dans son étude des modes de la domination, Max Weber souligne: «Sur le plan strictement psychologique la chaîne de causalité peut sembler différente, elle peut en particulier consister en une «suggestion » ou une «intuition». Mais cette distinction n'est pas utile, ici, à la formation des types de domination.», Economie et société. 1. Les catégories de la sociologie, Plon (Pocket), 1995 (1971), p. 288). Le lien entre pouvoir et domination est posé par M. Weber: «Le détenteur du pouvoir domine » (ibid., p. 303; voir la définition précise ibid., p. 95)

${ }^{36}$ L'infini est le lieu du pouvoir: il faut rapprocher cette problématique de l'analyse des Ménines de Vélasquez par Foucault (Les mots et les choses, op. cit.). 
pu se révolter - tuer le gardien ${ }^{37}$. Il ne le fait pas et se soumet ainsi à ce représentant de l'autorité. Si le pouvoir mis en acte dans cette légende se limitait à cela, il n'y aurait pas d'histoire. Car ce qui est essentiel, c'est que l'homme reste là devant la porte et que les années passent. Notons que cette obéissance est la première conséquence de la mécanique du pouvoir qu'il nous intéresse de décrire. Il faut que l'homme s'arrête devant la porte un instant, et l'acte de pouvoir traditionnel joue pour cela son rôle.

II. Une fois le passage «barré» par le garde, l'homme pourrait bien aller ailleurs. Pourquoi ne le fait-il pas? Refusons toute considération morale ou psychologique. C'est qu'il n'y a pas d'ailleurs dans cette parabole, comme il n'y a pas d'espace autour de ce dispositif minimal: la porte et son gardien. Le texte prosaïque, extrêmement précis et particulièrement lacunaire en regard d'une représentation réaliste, est si économe de ses éléments de description qu'il en «oublie» de donner quelque élément de spatialisation: la mécanique du pouvoir ne pourra être expliquée par un quadrillage de l'espace comme dans le Panoptique. L'indice majeur de la soumission de l'homme, c'est que n'ayant pas franchi la porte, il reste là, devant elle. Et cette soumission se mesure au passage du temps: toute cette vie d'homme consacrée à l'attente! Pour que ce mécanisme de pouvoir agisse, il ne suffit donc pas d'interdire: «l'attente à vie» est obtenue par un subterfuge qui tient à l'expérimentation originale du dispositif de vision exposée par le garde devant l'homme, puis à l'imitation partielle qu'en produit celui-ci durant toute sa vie: il observera attentivement la sentinelle, évitant le dispositif spéculaire que celle-ci lui offrait. Si l'interdit impose l'arrêt devant la porte, l'exemple et son imitation modèle toute une vie. Alors, la suggestion n'est pas seulement la variante d'un pouvoir hiérarchisé et traditionnel. Si l'interdit et l'exemple relèvent en définitive du même type de domination, lorsqu'ils sont articulés l'un à l'autre, comme dans la parabole, ils entrent dans un fonctionnement technologique du pouvoir: ils forment une machine dont les effets se combinent pour produire l'assujettissement. C'est parce que le pouvoir opère par cette machinerie que la dénonciation de la transcendance comme illusion, suggérée par le dispositif spéculaire, ne suffit pas pour échapper à la domination.

Or, à l'aune du temps qui passe, la place de l'homme ressemble fort à celle du gardien. Lui aussi demeure toute sa vie devant la porte. A considérer cet aspect de l'assujettissement certes non négligeable, puisqu'il s'agit de ce qui guide toute une vie d'homme, ils sont égaux ${ }^{38}$. Ils ne sont certes pas égaux devant la Loi, dont le garde est le représentant. Celui-ci pourra justifier le temps passé là, sa vie, par sa place en référence à la Loi. Ce n'est pas le cas de l'homme; mais, nous l'avons vu, ce dernier pourra revendiquer un espace de liberté dans l'usage qu'il fait du modèle spéculaire: il regarde le gardien se détournant de la porte. On pourrait formuler ainsi leur différence: le garde est sujet en tant qu'il est fondé par la Loi, qui justifie son pouvoir sur l'homme; ce dernier est sujet en tant qu'il exerce mini-

${ }^{37}$ L'assassinat du Père dans l'ordre traditionnel est encore un effet du pouvoir: sa transgression par le sang.

${ }^{38}$ C'est d'ailleurs une des pistes ouvertes par le commentaire du prêtre, mais sous l'angle du jugement, donc sous l'empire de la Loi et par rapport à un interdit. 
malement un choix, ce qui peut apparaître comme une compensation. Dans les deux cas, l'être sujet n'enlève rien à l'assujettissement qui se mesure au temps ${ }^{39}$.

Ce qui nous frappe est la surprésence du modèle archaïque du pouvoir; mais ce q'il faut voir est que ces différentes variantes du modèle traditionnel - parole de l'interdit ou du droit, dispositif de vision de la transcendance, autorité par l'exemple - sont les différentes articulations du pouvoir technologique.

III. Cependant, un troisième effet de la mécanique du pouvoir déployée par cette fable doit être retenu. Il ne suffit pas que l'homme obéisse à l'interdit; il ne suffit pas qu'il reste rivé à cette porte et à ce garde sa vie durant. Pour que l'assujettissement opère jusqu'au bout, il faut que cette vie, malgré la marge de liberté, soit une vie "perdue». Il faut que l'homme puisse mesurer que son choix conscient ou non d'ailleurs, cela nous intéresse peu ici - a été une erreur. La mécanique du pouvoir parachève l'assujettissement en transformant son choix minimal, sa petite marge de vie, en un échec. C'est un moment crucial, qui est d'autant plus marqué qu'il est le ressort du sentiment de tragique produit par la fable. L'assujettissement est bien de passer son temps devant la porte, mais c'est surtout de mesurer la valeur de ce temps à l'aune du premier modèle de pouvoir: lorsque la lumière apparaît, avec toute sa charge symbolique, le garde assène ses dernières paroles, qui relèvent de l'ordre archaïque: il dit à l'homme son droit singulier, droit que l'homme a manqué. Il est trop tard, et il s'est trompé.

Le retour de la Loi, même accompagné des indices de l'illusion, est essentiel: il permet de boucler le mécanisme. Mais l'importance de ce troisième temps ne tient pas à la puissance intrinsèque de la loi transcendante. Il est fonction de la place qui revient à la transcendance dans le processus global d'assujettissement: c'est-à-dire, après que l'homme a pu bénéficier d'une petite marge de liberté. C'est alors son choix, sa vie, qui sont jugés comme des erreurs, puisqu'il s'est détourné de la Lumière, ou qu'il n'a su entrer à temps. A l'horizon de son action se tient toujours la $\mathrm{Loi}^{40}$.

Le jugement et l'évaluation sont les ressorts du dernier mécanisme de la machine. Le dernier moment de l'assujettissement dépend donc du rôle qui est accordé à la loi: elle apparaît comme la justification ultime du comportement, le principe d'évaluation de l'action. Si l'on pense le pouvoir en le définissant à partir de la valeur que représente la Loi, comme y incitent les commentaires du prêtre et de $\mathrm{K}$, on reste dans la description du modèle archaïque. Tout commentaire qui vise au jugement en référence à cette valeur obéit à la machinerie de pouvoir de la fable. Ainsi se poursuit, dans le roman, la mécanique mise en scène dans «Devant la Loi».

Pourtant, si la Loi «fonctionne» effectivement comme le principe à partir duquel l'homme est jugé, elle n'est pas en tant que telle le garant du pouvoir

${ }^{39}$ Foucault joue effectivement sur ces deux termes: devenir sujet passe par l'assujettissement.

${ }^{40}$ La fable est entièrement placée sous le signe de l'erreur et du jugement de vérité: «—C'est sur la justice que tu te méprends, lui dit l'abbé, et il est dit de cette erreur dans les écrits qui précèdent la Loi : «Une sentinelle se tient postée devant la Loi (...)»», (op. cit., p. 263). Le prêtre raconte la parabole pour montrer que K se trompe sur la justice: il pousse le lecteur comme son auditeur à s'interroger sur la Vérité. 
exercé par la machinerie en son entier $^{41}$. La spécificité de «Devant la Loi » est de produire de l'assujettissement tout en intégrant la Loi comme une des pièces de la mécanique du pouvoir. Si elle est nécessaire à la formulation de l'interdit et de l'exemple, et si elle est désignée par le dispositif de vision premier et par la parole comme un élément indispensable, elle ne suffit pas à expliquer tout le processus. La mise en chaîne des formes de domination, qu'il faut distinguer de la simple répétition, donne la structure du pouvoir. En somme, le texte ne nous dit pas ce qu'est la Loi pas plus qu'il ne nous dit qu'elle n'est qu'une illusion. Il nous montre comment fonctionne le pouvoir, étant donné que l'un de ses rouages est ce qu'on appelle la Loi, construite comme une transcendance.

Comme Foucault l'analyse pour l'assujettissement moderne, le pouvoir passe ici par un agencement dont, paradoxalement, les figures archaïques sont les différentes éléments. On ne peut se contenter de décrire la loi transcendante et le pouvoir archaïque de la censure pour comprendre le fonctionnement de l'assujettissement dont il est question. Il ne suffit pas non plus de constater l'inadéquation de l'homme face à la Loi archaïque, car sa réaction est une adaptation qui prend place dans les différentes étapes à travers lesquelles agit la machine à pouvoir. L'inadéquation ne permet pas de penser en effet l'intégration de ce modèle traditionnel à une technologie du pouvoir dans laquelle les dispositifs de vision jouent un rôle important. La fable nous montre au contraire un fonctionnement parfaitement efficace où s'enchaînent plusieurs registres de l'exercice du pouvoir (interdit, suggestion par l'exemple, jugement référé à un sens, avec une adaptation de l'homme (imitation/appropriation) à ce qui lui est proposé.

Si nous soulignons la dimension technologique de ce pouvoir mis en scène par Kafka, ce qui en fait un pouvoir moderne dans le sens de Foucault, pouvons-nous pour autant l'assimiler au Panoptique, qui est devenu le parangon du pouvoir technologique? Nous l'avons vu, le quadrillage de l'espace qui permet de construire une machinerie de pouvoir caractéristique n'intervient pas dans la parabole. De plus, le dispositif de vision (porte plus sentinelle) qui participe à la mécanique de domination place le symbole du pouvoir à la place de l'objet du regard, et donc relève du modèle archaïque. Le dispositif de vision premier, dans la fable, est du côté du pouvoir traditionnel, faisant de la Loi, d'abord, et du gardien, ensuite, ce qu'il faut contempler. Par ailleurs, l'homme, et aussi le garde, sont constamment décrits comme des points de vue, quel que soit d'ailleurs leur usage du dispositif. Ils sont pourtant assujettis à titres divers. Le Panoptique, au contraire, présente une contrainte de définition que Foucault cerne précisément: il place le pouvoir là où est situé le regard.

Pourtant, dans la mesure où le panoptisme vise à constituer un système de contrôle, nous pouvons considérer que le résultat de la machinerie de «Devant la

${ }^{41}$ CF PLUS LOIN...C'est dans ce sens que va la lecture de Deleuze et Guattari: «La transcendance de la loi était machine abstraite, mais la loi n'existe que dans l'immanence de l'agencement machinique de la justice. Le Procès, c'est la mise en pièce de toute justification transcendantale. Il n'y a rien à juger dans le désir, le juge est lui-même tout entier pétri de désir. La justice est seulement le processus immanent du désir» (op. cit., p. 93). Nous rejoignons cette analyse. Cependant, notre analyse s'efforce néanmoins d'aborder l'«agencement» de pouvoir sans faire de la question du désir le ressort essentien de la machinerie, ce qui est le cas de l'analyse de Deleuze et Guttari, à lire absoluement en coninuation de L'anti.Edipe, ... 
loi » aboutit à ce résultat spécifique: l'assujettissement de l'homme et du garde qui les retient devant la porte fait de l'un l'observateur de l'autre, et réciproquement. Si la fable insiste sur le regard de l'homme, elle ne mentionne qu'une fois, nous l'avons vu, le garde en spectateur de son interlocuteur. Reste que le second niveau d'assujettissement, qui consiste à demeurer devant l'entrée de la Loi, équivaut à se tenir dans le face à face avec le gardien: le regard de l'un sur l'autre n'est jamais présenté comme une surveillance de l'interlocuteur; mais il a pour conséquence que chacun sait ce que l'autre fait. L'effet de cette pratique du dispositif premier - et qui consiste à se détourner de la Loi - est bien de transformer les deux sujets de regard en objet du regard de l'autre: leur relation est en somme «réciproquement panoptique». Etre là c'est bien être assujetti dans cette relation qui ne se définit plus tant par le pouvoir qu'accorde la Loi archaïque que par le contrôle qu'exercent l'un sur l'autre les deux hommes. Et ce contrôle s'effectue d'autant plus que l'homme existe comme sujet (de regard), qu'il peut croire à sa liberté de choix, et que le garde assume son autorité (qu'il tient de la loi ). L'effet panoptique se trouve décuplé par le maintien de la loi archaïque dans le système ainsi que de l'enchaînement des variantes du pouvoir qui s'y réfère.

S'il y a bien un fonctionnement panoptique, donc, il ne peut se comprendre à partir du dispositif de vision premier, le plus évident, la porte comme point de point de vue. Celui-ci distribue les positions de point de vue et d'objet selon le modèle archaïque. Ce n'est que par la machinerie du pouvoir que les personnages parviennent à des usages détournés de ce premier dispositif, ce qui implicitement induit un effet de panoptisme. Encore une fois, le pouvoir technologique apparaît dans l'articulation des usages et des variantes du pouvoir archaïque.

Quelle place historique doit-on accorder au processus d'assujettissement que déploie la parabole de Kafka? Nous ne répondrons certes pas ici à la question. Sans doute, le modèle devrait être confronté à des formes de pouvoir analogues ou concurrentes, dégagées à partir d'autres discours, tirés d'un corpus historiquement défini à travers plusieurs disciplines. Il faudrait, en somme, en appeler à une approche épistémologique des dispositifs de vision en relation avec la question du pouvoir, travail auquel Foucault nous invite.

Néanmoins, cette parabole démontre la nécessité de préciser toujours le lien entre point de vue construit et pouvoir. Il est nécessaire d'analyser tout dispositif de vision dans le détail de son fonctionnement car, nous l'avons vu, un dispositif qui renvoie a priori au pouvoir archaïque peut être agencé de manière à produire un assujettissement technologique: à partir d'un dispositif qui ne peut se comprendre à partir du Panoptique, nous avons été amenés pourtant à décrire du panoptisme. C'est que la machinerie du pouvoir ne dépend pas seulement d'une structure distribuant des places, elle est aussi fonction de la finalité de ce dispositif, c'est-à-dire de son usage social ou institutionnel, comme elle est définie par l'articulation interne des places de regard liées à des pratiques plurielles, notamment verbales, et dépendantes les unes autres.

Université de Lausanne 